\title{
DISCUTIR UM CASO DE GRAVIDEZ HETEROTÓPICA PARA EMPODERAMENTO DO PROFISSIONAL ENFERMEIRO
}

\author{
TO DISCUSS A CASE OF HETEROTOPIC PREGNANCY FOR \\ EMPOWERMENT OF THE PROFESSIONAL NURSE
}

\author{
Giovana Wachekowski ${ }^{1}$, Thaysi Carnet Figueiredo ${ }^{1}$, Alessandra Frizzo da Silva ${ }^{1}$ e \\ Lilian Zielke Hesler ${ }^{1}$ \\ ${ }^{1}$ Universidade Regional Integrada do Alto Uruguai e das Missóes (URI), Santo Ângelo, RS, \\ Brasil.
}

\section{RESUMO}

Objetivo: relatar um caso de gravidez heterotópica vivenciado por acadêmicas de enfermagem durante as atividades práticas da disciplina de enfermagem no cuidado à saúde da mulher. Método: trata-se de um estudo qualitativo, do tipo estudo de caso, frente a um caso de gravidez heterotópica. A coleta de dados ocorreu por meio de uma entrevista e os dados foram analisados por meio da análise de conteúdo das falas. Obteve-se a aprovação no comitê de ética em pesquisa da URI, campus Santo Ângelo, através do número 3.257.732 e respeitou os preceitos éticos da resolução 466/12. Resultados: a usuária apresentou intercorrências clinicas em sua segunda gestação de gemelares, decorrente de gravidez heterotópica, localizada na tuba uterina esquerda. Realizado salpingectomia da tuba uterina esquerda, conseguindo preservar a gestação uterina. Conclusáo: $\mathrm{O}$ enfermeiro precisa estar empoderado acerca de sinais e sintomas de complicaçóes que podem estar presentes durante uma gestação, como a gravidez heterotópica, tendo em vista que o mesmo realiza consultas de pré-natal, orientaçóes e trabalha com uma equipe multiprofissional. A escassez de trabalhos científicos voltados para a atuação do profissional enfermeiro frente a casos como estes, dificultou a discussão e o embasamento teórico voltados para a área.

Descritores: Gravidez Heterotópica; Gravidez Ectópica; Enfermagem; Cuidado Pré-Natal.

\section{ABSTRACT}

Objective: to report a case of heterotopic pregnancy experienced by nursing students during the activities of nursing discipline practices in women's health care. Method: this is a qualitative study, of the case study type, compared to a heterotopic pregnancy case. Data collection took place through an interview and the data were analyzed by analyzing the content of the statements. Obtained a research ethics committee from URI, Santo Ângelo campus, through number 3,257,732 and respected the ethical precepts of resolution 466/12. Results: the user presents clinical complications in the second 
twin pregnancy, due to heterotopic pregnancy, located in the left uterine tube. Salpingectomy of the left uterine tube was performed, managing to preserve the uterine pregnancy. Conclusion: The nurse needs to be involved with the signs and symptoms of complications that may be present during a pregnancy, such as a heterotopic pregnancy, considering that the same prenatal consultations, guidelines and work with a multidisciplinary team. The scarcity of scientific works aimed at the performance of the professional nurse in the face of cases like these, makes it difficult to discuss and theoretical grounding focused on an area.

Descriptors: Heterotopic pregnancy; Ectopic pregnancy; Nursing; Prenatal care.

\section{INTRODUÇÃO}

As gestações ocorrem após a implantação do blastocisto no endométrio da cavidade uterina. No entanto, devido a múltiplos fatores, podem ocorrer gestaçóes extrauterinas, isto é, quando a implantação do blastocisto se dá fora do útero, em sítios como a tuba uterina, colo uterino, cavidade abdominal e ovários, sendo denominada Gravidez Ectópica (GE). A maioria das GEs acontecem na tuba uterina, sendo responsável por 95 a $96 \%$ dos casos $^{1}$.

Entretanto, pode ocorrer o desenvolvimento simultâneo de uma gestação intrauterina e outra extra-uterina, definida como uma Gravidez Heterotópica $(\mathrm{GH})^{2}$. Os sintomas de Gravidez Heterotópica (GH) são caracterizados, em um primeiro momento, por dores abdominais intensas, mais precisamente na fossa ilíaca, e que evoluem rapidamente para náuseas, vômitos, episódios de lipotimia e distensão abdominal, além de hemorragias, se ocorrer o rompimento da tuba uterina ${ }^{3,4,2}$.

Alguns fatores podem elevar as chances de uma GH como, as cirurgias pélvicas anteriores, malformações uterinas, Doenças Inflamatórias Pélvicas (DIP), histórico de infertilidade, idade avançada, tabagismo e fertilização in vitro são os principais fatores ${ }^{5,1}$.

Todavia, mesmo com sintomas que evoluem para uma condição clínica grave de forma abrupta, a GH é considerada um caso raro, sendo em sua maioria, diagnosticada tardiamente e, em $80 \%$ dos casos, identificada somente após a rotura da gravidez ectópica ${ }^{6}$. Isto deve-se a baixa suspeita clínica de GE, devido à existência concomitante de uma gravidez intrauterina ${ }^{7,8}$. Entretanto, o atraso no diagnóstico da GE pode acarretar em complicaçóes para a saúde da gestante e para a gravidez intrauterina ${ }^{9,8}$.

Sendo assim, o profissional enfermeiro deve conhecer a GH, saber identificar seus sintomas e fatores de risco, tendo em vista que cabe ao mesmo realizar consultas de pré-natal de gestantes classificadas como de baixo risco ou de risco habitual, intercaladas com o profissional médico, a fim de assegurar uma assistência qualificada e integral ${ }^{10,11}$.

Neste sentido, a pesquisa se justifica na medida em que pretende explanar um caso de GH, uma complicação rara e com poucas publicaçóes recentes a respeito, principalmente por profissionais enfermeiros, fator que resulta em desconhecimento dos 
mesmos e repercute em diagnóstico tardio e risco de vida para a gestante e para a gravidez intrauterina.

Entende-se que, compartilhar este caso com enfermeiros e acadêmicos da área, pode auxiliar na reflexão e atualização dos mesmos, para que, durante a consulta de enfermagem, ao avaliar a gestante e às suas queixas, esteja apto a suspeitar, o mais precoce possível, de uma GH, realizando, juntamente com a equipe multiprofissional, discussóes e os devidos encaminhamentos, evitando maiores complicaçóes para a gestante e permitindo que a gestação intra-uterina seja mantida de forma sadia.

O objetivo deste estudo é relatar um caso de gravidez heterotópica vivenciado por acadêmicas de enfermagem durante as atividades práticas da disciplina de enfermagem no cuidado à saúde da mulher.

\section{MÉTODO}

Trata-se de um estudo de abordagem qualitativa, do tipo estudo de caso. A pesquisa qualitativa é centrada em dados que não são passíveis de quantificação, ou seja, nas relaçôes sociais, crenças, atitudes e valores, permitindo a construção ou aprimoramento de novos conceitos ${ }^{12}$. Já o estudo de caso analisa, tanto um único indivíduo, como grupos de pessoas, avaliando por que os mesmos se comportam ou pensam de um modo em $\operatorname{particular}^{13}$.

A participante do estudo foi uma gestante de sete semanas, que encontrava-se no pós-cirúrgico em decorrência de uma gravidez heterotópica. $\mathrm{O}$ primeiro contato com a gestante foi durante a atividade prática da disciplina de enfermagem no cuidado à saúde da mulher, realizado em uma Unidade Básica de Saúde (UBS), do município de Santo Ângelo/RS.

As acadêmicas de enfermagem e a professora do curso de graduação da Universidade Regional Integrada do Alto Uruguai e das Missóes - Campus Santo Ângelo/RS, juntamente com a Agente Comunitária de Saúde (ACS) realizaram uma visita domiciliar a essa gestante a pedido da enfermeira da unidade de saúde.

Após este primeiro contato, iniciou-se a construção de um referencial teórico, através das bases de dados Lilacs, Scielo e Biblioteca Virtual em Saúde, a fim de identificar estudos sobre a temática, originando um projeto de pesquisa sobre a temática. $\mathrm{O}$ projeto foi encaminhado ao Comitê de Ética em Pesquisa (CEP) da Universidade Regional Integrada do Alto Uruguai e das Missóes - Campus Santo Ângelo/RS, e foi aprovado sob número 3.257.732.

Após a aprovaçáo do projeto de pesquisa, a gestante foi convidada a participar do estudo e assinar o Termo de Consentimento Livre e Esclarecido (TCLE). Foi esclarecido 
a gestante sobre a sua livre participação na pesquisa, preservação do anonimato e respeito à liberdade de náo participar do estudo, e retirar-se do mesmo a qualquer momento.

A coleta de dados ocorreu na residência da gestante, por meio de agendamento prévio, sendo utilizado uma entrevista que incluía informaçóes acerca dos dados de identificação, antecedentes pessoais e gineco-obstétricos, histórico familiar, condições e hábitos de vida, história atual e análise de exames clínicos.

A análise dos dados foi realizada através da análise de conteúdo das falas, que trata-se de diversas técnicas, com procedimentos objetivos e organizados, auxiliando a averiguar e descrever os conteúdos das falas e/ou comunicaçóes ${ }^{14}$.

\section{APRESENTAÇÃO E RELATO DO CASO}

Gestante, 23 anos, tipo sanguíneo $\mathrm{B}+$, histórico gestacional de duas gestações, considerando a atual, um parto cesáreo, sem antecedentes de abortos ou complicaçóes na gestação anterior. Saudável, sem histórico de tabagismo, etilismo ou drogas, doenças prévias, cirurgias, de complicaçóes ginecológicas ou gestaçóes gemelares e/ou ectópicas na família. As duas gestações foram obtidas por meio da concepção espontânea. A primeira gestação ocorreu há seis anos, com parto do tipo cesárea e sem intercorrências no prénatal, parto e pós-parto.

No entanto, a segunda gestação trouxe sintomas diferentes. No dia 16 de novembro de 2017, quando a mesma encontrava-se com sete semanas de gestação, iniciaram dores leves, tipo cólicas menstruais, localizadas precisamente na fossa ilíaca esquerda, que foram se intensificando conforme o passar das horas.

Neste mesmo dia realizou uma consulta médica, onde na ecografia, que segundo a mesma, teve como foco somente a gravidez intrauterina, não evidenciava alteraçóes. Os próximos três dias foram de dores intensas, sem melhora do quadro clínico, passando por mais duas consultas, ambas com o mesmo resultado das anteriores.

No dia 20 de novembro a paciente deu entrada na emergência do hospital de referência de seu município com êmese, dores abdominais intensas, palidez cutânea, abdômen distendido e episódios de lipotimias. Ao realizar uma nova ecografia, foi identificado líquido na região abdominal, sendo encaminhada imediatamente para uma cirurgia de urgência. Entretanto, segundo a mesma, a cirurgia foi realizada sem um diagnóstico preciso, havendo apenas, uma suspeita de apendicite aguda ou cálculos renais. Durante a cirurgia aberta, houve o diagnóstico de uma gravidez heterotópica, isto é, além da gravidez intrauterina, ela estava com uma gravidez ectópica, localizada na tuba uterina esquerda.

Foi realizado então, uma salpingectomia a esquerda. A gestante permaneceu internada por uma semana, recebendo infusão de sangue, devido a intensa hemorragia, 
com boa recuperação do quadro clínico e com a gravidez intrauterina mantida, sem alteraçóes.

A gravidez evoluiu até a 38 a semana de gestação, onde então, no dia 28 de julho de 2018, o bebê nasceu de parto cesárea, sem complicaçóes, pesando 3.395 e medindo $49 \mathrm{~cm}$ de comprimento. Foi possível acompanhar o pós-parto imediato da puérpera, nas atividades práticas da disciplina de Enfermagem no Cuidado a Saúde da Mulher, também em âmbito hospitalar. Onde identificou-se que, tanto a puérpera, quanto o recém-nascido, estavam saudáveis e sem alteraçóes.

\section{DISCUSSÃO}

Existem alguns fatores que elevam as chances de uma Gravidez Heterotópica e Gravidez Ectópica que são as doenças inflamatórias pélvicas, malformaçóes uterinas, cirurgias pélvicas anteriores, histórico de infertilidade, idade avançada, tabagismo e fertilização in vitro ${ }^{5,1}$. Todavia, a gestante deste caso descrito não apresenta nenhum dos fatores de risco para GH ou GE.

Os sintomas apresentados pela gestante, são típicos de GE localizada nas tubas uterinas, sendo caracterizada, em um primeiro momento, por dores abdominais intensas, tipo cólicas, mais precisamente na fossa ilíaca, e que evoluem rapidamente para náuseas, vômitos, episódios de lipotimia e distensão abdominal, além de hemorragias, se a tuba uterina já tiver rompido ${ }^{3,4,2}$. Entretanto, mesmo com sintomas típicos e com o agravamento rápido do quadro clínico, a GH pode ser de difícil diagnóstico inicialmente, podendo ser confundida, por exemplo, com uma ameaça de aborto da gravidez intrauterina, apendicite aguda, diverticulite e cálculos renais ${ }^{15,8}$, como foi o caso da gestante deste estudo.

Primeiras hipóteses diagnósticas errôneas e a demora de dias ou semanas para um diagnóstico concreto de $\mathrm{GH}$, bem como a necessidade de uma intervenção cirúrgica para o reconhecimento de uma GH não são incomuns e já foram relatadas em outros estudos de $\operatorname{casos}^{2,1}$. Um dos fatores que podem contribuir para que o diagnóstico de GH seja dado somente no momento da intervenção cirúrgica, após a exacerbação dos sintomas, é a presença da gravidez intrauterina associada a hemorragia interna ${ }^{16,2}$.

As intervençóes nestes casos, devem ser as menos invasivas possíveis, a fim de preservar a gravidez intrauterina, mantendo uma mínima manipulação uterina. Neste sentido, a salpingectomia por laparoscopia vem sendo o procedimento cirúrgico mais adotado, segundo alguns estudos recentes ${ }^{2,17}$. Quanto à fertilidade, estudo de 2015 identificou não haver diferença entre a salpingostomia e a salpingectomia ${ }^{2}$.

Após ser diagnosticada uma gestação intrauterina, é necessário atentar para as queixas da gestante em todas as consultas de pré-natal, realizadas por enfermeiros 
e médicos, e explorar regióes anexiais, principalmente na primeira ultrassonografia da gestação, por volta das 7 ou 8 semanas, mesmo que não exista fatores de risco para $\mathrm{GH}^{8}$.

Além disso, o enfermeiro deve observar os sinais e sintomas relatados pela gestante durante as consultas de pré-natal na unidade e à domicílio, além de fazer a busca ativa da mesma, mesmo que náo esteja realizando o pré-natal na unidade, a fim de, em conjunto com a equipe multiprofissional, estar discutindo o caso, elaborando o plano de cuidados e possíveis encaminhamentos e exames.

\section{CONSIDERAÇÓES FINAIS}

A realização deste estudo de caso foi de extrema relevância para o aprimoramento dos conhecimentos pré existentes, para o desenvolvimento de um olhar crítico-reflexivo acerca dos sinais e sintomas que podem estar presentes durante uma gestação intrauterina e para visualização da importância da atuação de um profissional enfermeiro atualizado e bem preparado nas consultas de pré-natal.

A escassez de trabalhos científicos voltados para a atuação do profissional enfermeiro frente a casos como estes, dificultou a discussão e o embasamento teórico voltados para a área. Neste sentido, ressalta-se a importância de mais estudos sobre a gravidez heterotópica, principalmente no que se refere a atuaçáo profissional enfermeiro frente a mesma.

\section{REFERÊNCIAS}

1- Martins-costa SH, Ramos JGL, Magalhães JA, Passos EP, Freitas F. Rotinas em obstetrícia. $7^{\text {a }}$ ed. Porto Alegre: Artmed, 2017.

2- Magalhães M, Areia A, Franco S, Fonseca E, Moura P. Misdiagnosis and delayed diagnosis for spontaneous heterotopic pregnancy: a case report. ActaActa Obstet Ginecol Port 2015; 9(4):293-296. Acesso em: 29 out 2019. Disponível em: http:// www.fspog.com/fotos/editor2/04-sup cc 14-00014.pdf.

3- Barbosa CP, Campoi GM, Oliveira KM, Santos ME, Fasano PR, Silva PDV, et al. Concomitância de gestação tópica e ectópica. Ciências da saúde do ABC. 2004; 29 (1). Acesso em: 20 jan 2019. Disponível em: http://bases.bireme.br/cgibin/wxislind.exe/ iah/online/?IsisScript=iah/iah.xis\&src=google\&base=LILACS\&lang=p\&nextAction=ln $\mathrm{k} \&$ exprSearch=457904\&indexSearch=ID.

4- Pizzato FB, Juliano HV, Fialho JC, Lino TH, Couri BM, Zorrón R. Videocirurgia na Prenhez Ectópica Rota com Prenhez Tópica Concomitante. Rev bras videocir 2004; 2(3): 148-151. Acesso em: 28 out 2019. Disponível em: https://www.sobracil.org.br/ revista/rv020203/rbvc020203 148.pdf. 
5- Clayton HB, Schieve LA, Peterson HB, Jamieson DJ, Reynolds MA, Wright VC. A comparison of heterotopic and intrauterine-only pregnancy outcomes after assisted reproductive technologies in the United States from 1999 to 2002. Fertil Steril. 2007; 87(2): 303-9. Acesso em: 28 out 2019. Acesso em: https://www.ncbi.nlm.nih.gov/ pubmed/17113092.

6- Corraliza V, Vicandi F, Granados MA. Embarazo heterotópico espontáneo presentado como abdomen agudo. Clin Invest Ginecol Obstet. 2002; 29 (9): 349-350. Acesso em: 7 mar 2019. Disponível em: https://www.elsevier.es/es-revista-clinica-einvestigacion-ginecologia-obstetricia-7-articulo-embarazo-heterotopico-espontaneopresentado-como-S0210573X02772109.

7- Ludwig M, Kaisi M, Bauer O, Diedrich K. Heterotopic pregnancy in a spontaneous cycle: do not forget about it. Eur J Obstet Gynecol Reprod Biol. 1999; 87:91103. Acesso em: 14 out 2019. Disponível em: https://www.ncbi.nlm.nih.gov/ pubmed/10579623.

8- Guimarães AC et al. Spontaneous Heterotopic Triplet Pregnancy with a Two Viable Intrauterine Embryos and an Ectopic One with Right Tubal Rupture. Rev Bras Ginecol Obstet. 2019; 41 (4); p. 268-272. Acesso em: 29 out 2019. Disponível em: 10.1055/s0039-1683910.

9- Franke C, Rohrborn A, Thiele H, Glatz J. Combined intrauterine and extrauterine gestation. A rare cause of acute abdominal pain. Arch Gynecol Obstet. 2001; 265(1):51-2. Acesso em: 14 out 2019. Disponível em: https://www.ncbi.nlm.nih.gov/ pubmed/11327095.

10- Brasil Ministério da Saúde. Secretaria de Atenção à Saúde. Departamento de Atenção Básica. Atenção ao pré-natal de baixo risco. Brasília, DF: Ministério da Saúde, 2012. Acesso em: 10 jun 2019. Disponível em: Disponível em: http://bvsms.saude.gov. br/bvs/publicacoes/cadernos atencao basica 32 prenatal.pdf.

11- Cardoso MD, Ribeiro CMS, Oliveira IB, Andrade PMC, Santos TMB. Perceptions of pregnant women about the organization of the service/assistance in prenatal low risk in Recife. Revista de Pesquisa, Cuidado é Fundamental online. 2016; 8 (4): 501724. Acesso em: 7 mar 2019. Disponível em: http://www.seer.unirio.br/index.php/ cuidadofundamental/article/view/4941/pdf 1

12- Minayo MCS. Pesquisa Social. Teoria, método e criatividade. 18 ed. Petrópolis: Vozes, 2001.

13- Polit DF, Beck CT. Fundamentos da pesquisa em enfermagem: avaliação de evidências para a prática de enfermagem. 7ª ed. Porto Alegre: ArtMed; 2011.

14- Bardin L. Análise de Conteúdo. 4ª ed. Lisboa: Portugal; 2009. 
15- Rezende JM, Montenegro CAB. Obstetrícia fundamental. Prenhez ectópica. $8^{\mathrm{a}}$ ed. Rio de Janeiro: Guanabara Koogan: 1999.

16- Sohail S. Hemorrhagic corpus luteum mimicking heterotopic pregnancy. J Coll Physicians Surg Pak. 2005; 15:180-181. Acesso em: 19 fev 2019. Disponível em: https://www.ncbi.nlm.nih.gov/pubmed/15808104.

17- Reis LDO, Leite FC, Reis CFD, Costa AP, Araújo WJB. Spontaneous Heterotopic Triplet Pregnancy with a Two Viable Intrauterine Embryos and an Ectopic One with Right Tubal Rupture. Rev Bras Ginecol Obstet. 2019; 41 (4): 268-272. Acesso em: 26 maio 2020. Disponível em: http://dx.doi.org/10.1055/s-0039-1683910.

Autor Correspondente: Giovana Wachekowski ${ }^{1}$ E-mail: giovanawachekowski@outlook.com

Recebido em: 2019-10-31

Aprovado: 2020-06-26 